\title{
Chrysomelidial in the Opisthonotal Glands of the Oribatid Mite, Oribotritia berlesei
}

\author{
Günther Raspotnig • Rene Kaiser • \\ Edith Stabentheiner • Hans-Jörg Leis
}

Received: 26 February 2008 /Revised: 23 April 2008 / Accepted: 20 May 2008 /Published online: 10 July 2008

(C) The Author(s) 2008

\begin{abstract}
Gas chromatographic-mass spectrometric analyses of whole body extracts of Oribotritia berlesei, a largesized soil-dwelling oribatid mite, revealed a consistent chemical pattern of ten components, probably originating from the well-developed opisthonotal glands. The three major components of the extract were the iridoid monoterpene, $(3 S, 8 S)$-chrysomelidial (about $45 \%$ of the extract), the unsaturated hydrocarbon 6,9-heptadecadiene, and the diterpene $\beta$-springene (the latter two, each about $20-25 \%$ of the extract). The remaining minor components (together about $10 \%$ of the extract) included a series of hydrocarbons (tridecene, tridecane, pentadecene, pentadecane, 8-heptadecene, and heptadecane) and the tentatively identified 9,17-octadecadienal. In contrast, analysis of juveniles showed only two compounds, namely a 2:1 mixture of $(3 S, 8 S)$ chrysomelidial and its epimer, epi-chrysomelidial $(3 S, 8 R$ chrysomelidial). Unexpectedly, neither adult nor juvenile secretions contained the so-called astigmatid compounds, which are considered characteristic of secretions of oribatids above moderately derived Mixonomata. The chrysomelidials, as well as $\beta$-springene and octadecadienal, are newly
\end{abstract}

\footnotetext{
G. Raspotnig $(\bowtie) \cdot$ R. Kaiser

Institute of Zoology, Karl-Franzens-University,

Universitätsplatz 2,

8010 Graz, Austria

e-mail: guenther.raspotnig@uni-graz.at

G. Raspotnig $\cdot$ R. Kaiser $\cdot$ H.-J. Leis

Department of Biochemical Analysis and Mass Spectrometry,

Medical University, University Children's Hospital,

Auenbruggerplatz 30,

8036 Graz, Austria

E. Stabentheiner

Institute of Plant Sciences, Plant Physiology,

Schubertstrasse 51,

8010 Graz, Austria
}

identified compounds in the opisthonotal glands of oribatid mites and have chemotaxonomic potential for this group. This is the first instance of finding chrysomelidials outside the Coleoptera.

Keywords Iridoid monoterpene - Oil gland · Chemosystematics $\cdot \beta$-Springene $\cdot$ Oribatida

\section{Introduction}

The largest and most conspicuous exocrine system in the acariform mite suborders Oribatida and Astigmata is the paired opisthonotal glands (syn. oil glands). These glands, according to a current and widely accepted hypothesis, evolved only once within ancient Oribatida (after the nearbasal oribatid groups, Palaeosomata and Enarthronota, had split-off), and are considered characteristic of the four more-derived oribatid groups, Parhyposomata, Mixonomata, Desmonomata, Brachypylina, and also of astigmatid mites. The presence of opisthonotal glands not only constitutes a major argument for a proposed monophyletic unit of socalled glandulate Oribatida, including Astigmata (Norton 1998), but also provides evidence for the evolutionary origin of astigmatid mites in an ancient opisthonotal glandbearing oribatid group.

In addition to the presence or absence of opisthonotal glands, the secretions of these glands provide phylogenetic information as they include a rich variety of hydrocarbons, terpenes, aromatics, and alkaloids in species-specific or group-specific patterns. These secretions, derived from a homologous gland system, allow the tracing of evolutionary lineages from near-basal to more-derived glandulate Oribatida. Apart from the well-studied opisthonotal gland secretions of Astigmata (Kuwahara 2004), the secretions of a selection 
of species of several major groups of glandulate oribatids have been investigated, including representatives of Parhyposomata (Sakata and Norton 2001), a few species of Mixonomata and Desmonomata (Sakata et al. 1995, 2003; Raspotnig et al. 2001, 2005a, b; Shimano et al. 2002; Sakata and Norton 2003), and also examples of Brachypylina (Takada et al. 2005; Saporito et al. 2007). While Parhyposomata produce phenols and naphthols, the socalled astigmatid compounds (sensu Sakata and Norton 2001) evolved within ancient mixonomatans, and hence, are possibly found in all the above groups. These astigmatid compounds comprise a set of terpenes and aromatics, namely neral, geranial, neryl formate, 2-hydroxy-6-methylbenzaldehyde, and $\gamma$-acaridial. The distribution of astigmatid compounds in oribatids is of special interest since these compounds not only provide chemical support for the evolutionary origin of astigmatid mites within ancient Oribatida (Norton 1998), but also define a presumably monophyletic subunit within glandulate Oribatida, the “astigmatid compounds-bearing" oribatids (Raspotnig 2006).

Several chemotaxonomic studies that examined these presumptive astigmatid compound-bearing groups have been undertaken and have yielded a fragmented picture of the distribution of astigmatid compounds. These compounds are difficult to detect in the more derived groups of Desmonomata (apart from Trhypochthoniidae) and generally in Brachypylina, suggesting that, in these groups, astigmatid compounds have been prone to evolutionary reduction and replacement. In some cases, desmonomatan and brachypyline species are chemically "dimorphic", with astigmatid compounds present only in juveniles and completely replaced in adults by novel compounds such as toxic alkaloids (Takada et al. 2005; Saporito et al. 2007). In contrast, the full (or nearly full) set of astigmatid compounds seems to characterize highly derived mixonomatans, low to moderately derived representatives of Desmonomata, and Astigmata. Specifically, these compounds have been detected in opisthonotal gland secretions of Collohmannia gigantea (Raspotnig et al. 2001), in all Trhypochthoniidae so far investigated (e.g., Sakata et al. 1995, 2003), and in astigmatid mites (e.g., Kuwahara 2004). However, there are large gaps in our knowledge of the distribution of astigmatid compounds: the most conspicuous gap being in the Euphthiracaroidea, a speciose group of box mites whose opisthonotal gland chemistry has yet to be investigated. Euphthiracaroids are considered a group of highly derived mixonomatans that, together with phthiracaroids, constitute a possible sister group of Collohmannia. Thus, euphthiracaroids are likely candidates for producing astigmatid compounds. The study of the chemistry of opisthonotal glands in euphthiracaroids is important not only to further our understanding of the evolution of astigmatid compounds, but also because this group is a crucial link for tracing a possible evolutionary lineage from Oribatida to Astigmata. With respect to the latter, some (not all!) recent molecular studies (e.g., Murell et al. 2005; Domes et al. 2007) conflict with morphological data. Therefore, studying the opisthonotal gland chemistry may resolve this problem.

In the present paper, we provide the first chemical analysis of the opisthonotal gland secretion of Oribotritia berlesei, a large-sized oribatid mite from the near-basal euphthiracaroid family Oribotritiidae.

\section{Methods and Materials}

Mites and Mite Extracts Specimens of $O$. berlesei (Michael 1898) (mixonomatan Oribatida: Euphthiracaroidea, Oribotritiidae) were collected from the litter and fermentation layer of a mixed forest near Ferlach (Carinthia, Austria). Individuals were collected by hand or extracted from soil samples by using Berlese-Tullgren funnels. In all, 35 adults (all in the course of one collection in September 2006) were used for chemical analyses, five adults were used for scanning electron microscopy, and 30 adults were transferred to Petri dishes (equipped with Plaster of Paris and dead wood) and kept in the dark for a period of about 12 months either at room temperature (one Petri dish) or at $10^{\circ} \mathrm{C}$ (second Petri dish), respectively. Individuals of both Petri dishes laid eggs, and the offspring were reared to deutonymphal and tritonymphal instars. Since immatures are burrowers in dead wood, pieces of dead wood (provided in the rearing dishes) were checked for immatures at weekly intervals. Ten deutonymphs and eight tritonymphs were used in the chemical studies.

Extracts (containing opisthonotal gland secretions) of adults were prepared by immersing freshly collected, living, individuals in hexane (one individual per $100 \mu \mathrm{l}$ ) for $30 \mathrm{~min}$ (Raspotnig et al. 2001, 2005a, b). For juveniles, individual and pooled extracts (containing two to six individuals) were prepared similarly. The extracts were used without further cleanup.

Chemical Analysis: Gas Chromatography-Mass Spectrometry A trace gas chromatograph (GC) coupled to a Voyager mass spectrometer (MS; both from Thermo, Vienna, Austria) and equipped with a ZB-5MS fused silica capillary column $(30 \mathrm{~m} \times 0.25 \mathrm{~mm}$ i.d., $0.25 \mu \mathrm{m}$ film thickness, Phenomenex, Germany) was used for the analyses. Injection was splitless with helium (at a constant flow rate of $1.5 \mathrm{ml} \mathrm{min}^{-1}$ ) as a carrier gas. The column temperature was programmed from $50^{\circ} \mathrm{C}$ (held for $1 \mathrm{~min}$ ) to $200^{\circ} \mathrm{C}$ at $10^{\circ} \mathrm{C} \mathrm{min}^{-1}$, and then to $300^{\circ} \mathrm{C}$ at $15^{\circ} \mathrm{C} \mathrm{min}^{-1}$. The ion source of the mass spectrometer and the transfer line were kept at $150^{\circ} \mathrm{C}$ and $310^{\circ} \mathrm{C}$, respectively. Electron impact (EI) spectra were recorded at $70 \mathrm{eV}$. 
Derivatization, Syntheses, Reference Compounds, and Other Chemicals Tri-, penta-, and heptadecane, as well as citral (60\% geranial, $40 \%$ neral) and selenium dioxide for the preparation of oxocitral (see Bellesia et al. 1986), were purchased from Aldrich (Vienna, Austria). For determination of double bonds in the heptadecadiene, dimethyl-disulfide (DMDS) adducts were generated according to the method described by Vincenti et al. (1987). The opisthonotal gland secretion of Platynothrus peltifer (Raspotnig et al. 2005b) was used as a natural source for 6,9-heptadecadiene and 8heptadecene for chromatographic comparisons. In order to clarify further the structure of chrysomelidial, the $O$-methyloxime derivatives were prepared with methoxylamine hydrochloride (MOX.HCl; 2\% in pyridine): $200 \mu \mathrm{l}$ of MOX was added to $50 \mu \mathrm{l}$ of extract and left at $70^{\circ} \mathrm{C}$ for $1 \mathrm{~h}$. Products were cleaned in $\mathrm{H}_{2} \mathrm{O}$, extracted with hexane, and analyzed. Stereoisomers of chrysomelidial and dehydroiridodial were synthesized according to Bellesia et al. (1986). Secretions from eversible defense glands of larvae of the chrysomelid beetles, Gastrophya viridula and Plagiodera versicolora (collected in the surroundings of Graz, Austria), known to contain natural $(3 S, 8 S)$-chrysomelidial (e.g., Pasteels et al. 1982), were collected on small filter paper pieces, extracted in hexane (one individual in $100 \mu \mathrm{l}$ ), and used as sources for authentic reference compounds. Hexane extracts of fresh leaves of Actinidia polygama (obtained from the botanical garden in Tübingen, Germany) were taken as a natural source for $(3 R, 8 S)$-dehydroiridodial (Yoshihara et al. 1978).

Scanning Electron Microscopy Air-dried mites were mounted on aluminum stubs, sputtercoated (AGAR sputtercoater, Gröpl, Tulln, Austria), and examined with a
Philips XL30 ESEM (Philips/FEI, Vienna, Austria) at high vacuum mode and $20 \mathrm{kV}$ accelerating voltage.

\section{Results}

Extracts of Adult $O$. berlesei A total of ten components (peaks a-j) were detected in extracts of adult $O$. berlese $i$ (Fig. 1a). The main component in extracts, component c ( $\mathrm{RT}=13.36 \mathrm{~min}$; ca. $45 \%$ of the total extract, based on peak areas) had an EI fragmentation pattern resembling a methylcyclopentene monoterpene (Fig. 2a), with a molecular ion at $\mathrm{m} / \mathrm{z} 166(7)$ and characteristic fragments at $\mathrm{m} / \mathrm{z} 151$ (6), 148 (46), 138 (25), 133 (9), 123 (15), 120 (12), 119 (10), 109 (49), 108 (40), 105 (30), 95 (19), 91 (27), 81 (100), 79 (78), and 77 (38). The spectrum was consistent with that of chrysomelidial from the literature (e.g., Blum et al. 1978; Oldham et al. 1996) and with a compound from the NIST library ("2-formyl- $\alpha, 3$-dimethyl-2-cyclopentene-1-acetaldehyde"; CAS no. 75332-42-2: "2-methyl-5(1-methyl-2oxoethyl)-1-cyclopentene-1-carbaldehyde"). In order to prove the dialdehyde structure, we generated $O$-methyloxime derivatives, resulting in the elimination of peak $\mathrm{c}$ and the formation of two new peaks, $c^{\prime}$ and $c^{\prime \prime}$, with longer retention times $(\mathrm{RT}=14.92$ and $15.11 \mathrm{~min})$. The two compounds had identical mass spectra, with molecular ions at $\mathrm{m} / z 224$ (4), and fragment ions at $\mathrm{m} / z 193$ (13), 178 (22), 146 (25), 138 (100), 107 (38), 106 (95), 91 (18), 87 (21), 79 (47), and 77 (29). This pattern indicated $O$-methyloximation of compound c, forming two isomeric dimethyloximes, which confirmed the proposed dialdehydic structure (addition of two $O$-methyl-oxime groups to compound $\mathrm{c}=$ 166 plus $2 \times 29=$ new molecular ion at $m / z 224$ ).
Fig. 1 Typical mass chromatograms of extracts of Oribotritia berlesei: a adult individual. b Deutonymph. Peaks $a$ (tridecene), $b$ (tridecane), $c_{l}$ ("epi-chrysomelidial" $=3 S, 8 R$ chrysomelidial), $c$ ("chrysomelidial" $=3 S, 8 S$ chrysomelidial), $d$ (pentadecene), $e$ (pentadecane), $f$ (6,9-heptadecadiene), $g$ (8-heptadecene), $h$ (heptadecane), $i$ (tentatively, $\beta$-springene), $j$ (tentatively, 9,17-octadecadienal)

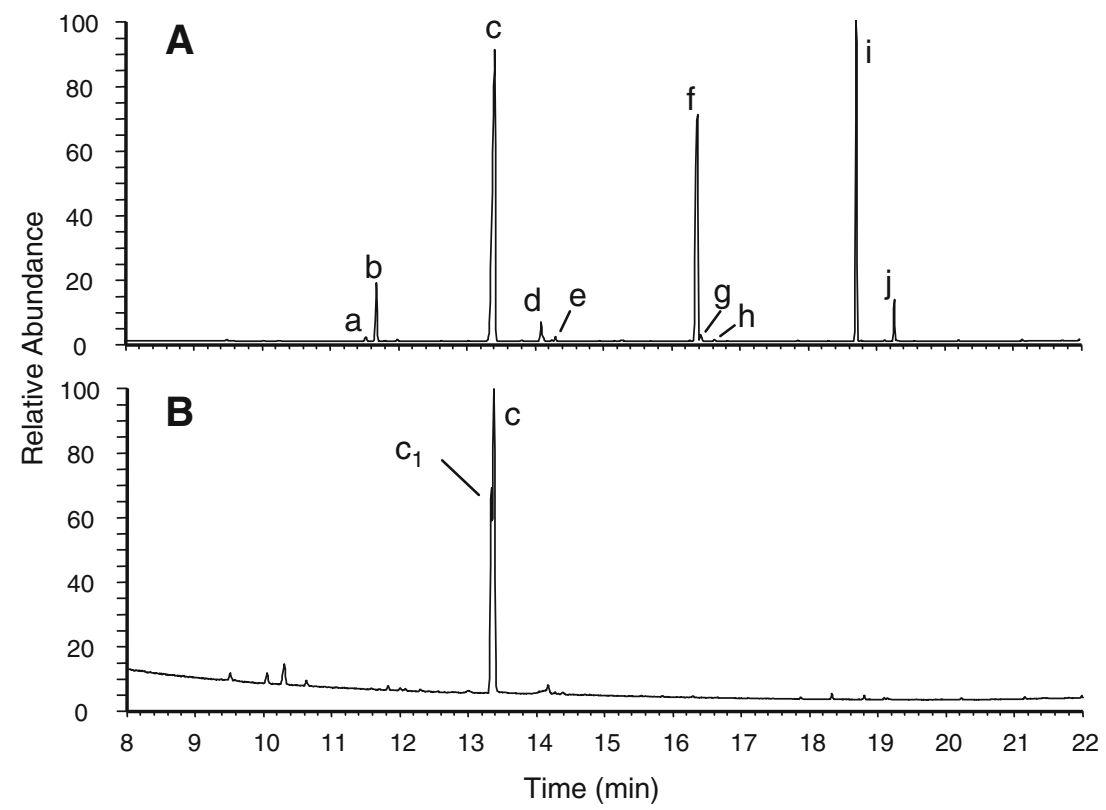


Fig. 2 Electron impact mass spectra of chrysomelidials in extracts of Oribotria berlesei. a Compound c, tentatively identified as $(3 S, 8 S)$ chrysomelidial. b Compound $\mathrm{c}_{1}$, tentatively identified as epi-chrysomelidial $=(3 S, 8 R)$ chrysomelidial. Note the differences in relative intensities of ions at $m / z 148\left(\mathrm{M}^{+}-\mathrm{H}_{2} \mathrm{O}\right)$ and $m / z 138\left(\mathrm{M}^{+}-\mathrm{CO}\right)$. Further diagnostic fragments are assigned to: $\mathrm{M}^{+}-\mathrm{H}_{2} \mathrm{O}-\mathrm{CO}(\mathrm{m} / \mathrm{z}$ 120); $\mathrm{C}_{7} \mathrm{H}_{9} \mathrm{O}^{+}$(m/z 109), $\mathrm{C}_{7} \mathrm{H}_{7}^{+}$ ( $\mathrm{m} / \mathrm{z}$ 91), and $\mathrm{C}_{6} \mathrm{H}_{9}{ }^{+}(\mathrm{m} / \mathrm{z}$ 81: base peak $=$ the methylcyclopentene moiety)
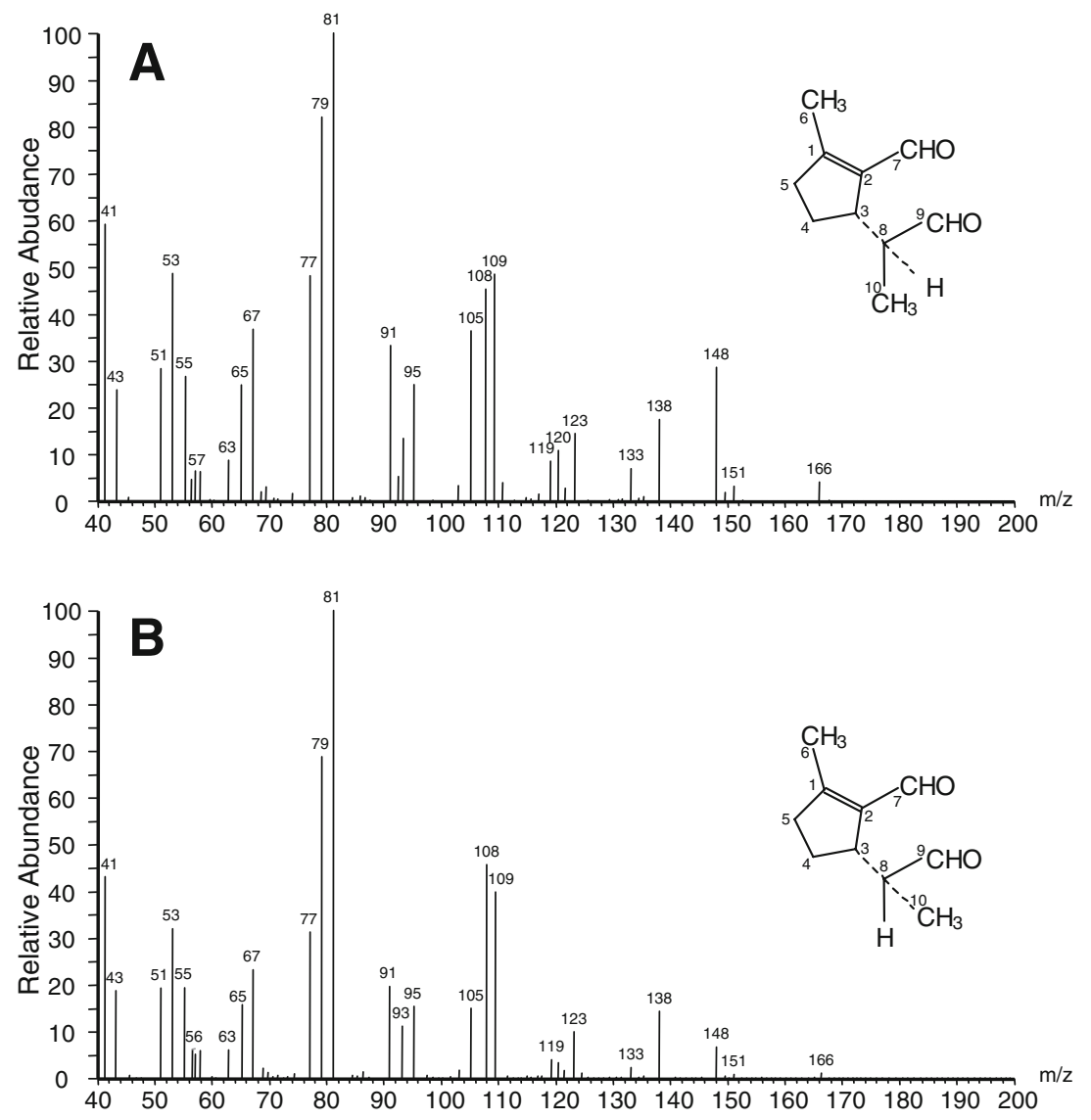

For a comparison of compound $\mathrm{c}$ to authentic chrysomelidial and its characterization, we used (1) a synthetic mixture of chrysomelidial-stereoisomers, and (2) naturally occurring $(3 S, 8 S)$-chrysomelidial. The synthetic mixture was assumed to contain four stereoisomers of chrysomelidial and dehydroiridodial (according to Bellesia et al. 1986), namely two diastereomeric pairs of enantiomers, i.e., $(3 S, 8 S)$-chrysomelidial, $(3 R, 8 R)$-dehydroiridodial, and $(3 S, 8 R)$-chrysomelidial (= epi-chrysomelidial), $(3 R, 8 S)$-dehydroiridodial. However, the mixture exhibited only two, poorly separable peaks at $\mathrm{RT}=13.32$ and $13.36 \mathrm{~min}$, each peak corresponding to one pair of enantiomers, with single enantiomers not separable under the chromatographic conditions. The later-eluting peak of the mixture corresponded to compound $\mathrm{c}$ from the Oribotritia extract, with respect to both retention time and EI fragmentation pattern. Larval secretions of the leaf beetle Gastrophysa viridula (see "Methods and Materials"), contain mainly $(3 S, 8 S)$-chrysomelidial ("original chrysomelidial"). Analysis of this secretion showed only one abundant component that eluted at $\mathrm{RT}=13.36 \mathrm{~min}$, corresponding to the later-eluting peak of the synthetic mixture and to compound $\mathrm{c}$ of the mite extracts. Thus, the later-eluting peak of the synthetic mixture was assigned as either $(3 S, 8 S)$ chrysomelidial or its enantiomer, $(3 R, 8 R)$-dehydroiridodial. Since dehydroiridodials are considered of plant origin only (see "Discussion"), compound c was tentatively identified as $(3 S, 8 S)$-chrysomelidial.

The mass spectrum of peak $f(\mathrm{RT}=16.36 \mathrm{~min})$, the second most abundant compound in extracts (about $25 \%$ of total), suggested a doubly unsaturated C17-hydrocarbon, based on a molecular ion at $\mathrm{m} / \mathrm{z} 236$ and a series of unsaturated hydrocarbon fragments. The DMDS adduct of the heptadecadiene showed a molecular ion at $\mathrm{m} / \mathrm{z} 362$ and diagnostic ions at $m / z 231,203,183,159,155$ (base peak), and 131, consistent with 6,9-heptadecadiene (see Raspotnig et al. 2005b). Compound i ( $R T=18.70 \mathrm{~min}$; ca. $20 \%$ of the total) showed a terpene fragmentation pattern with a molecular ion at $m / z 272$ (4) and further $m / z$ of 257 (3), 229 (4), 203 (5), 187 (10), 161 (17), 133 (31), 120 (15), 119 (17), 107 (20), 93 (50), 81 (37), 69 (100), 55 (16), 53 (17), and 41 (59). The compound was tentatively identified as $\beta$ springene on the basis of mass spectral comparison to $\beta$ springene spectra from literature and from the NIST library. The remaining peaks a, b, d, e, g, and h, all of them minor components, appeared to be a series of hydrocarbons, with compounds b $(\mathrm{RT}=11.69)$, e $(\mathrm{RT}=14.32 \mathrm{~min})$, and $\mathrm{h}(\mathrm{RT}=$ $16.65 \mathrm{~min})$ exhibiting the spectra of saturated $n$-alkanes with molecular ions at $m / z 184,212$, and 240, respectively. The compounds were identified as tri-, penta-, and heptadecane by comparison of retention times to authentic 
standards. Compounds a $(\mathrm{RT}=11.54), \mathrm{d}(\mathrm{RT}=14.10 \mathrm{~min})$, and $\mathrm{g}(\mathrm{RT}=16.44 \mathrm{~min})$ had mass spectra of monounsaturated analogs, with molecular ions at $\mathrm{m} / \mathrm{z} 182,210$, and 238, corresponding to tri-, penta-, and heptadecene. The position of the double bonds was not determined because, due to their low amounts, the compounds were not recovered after DMDS derivatization. The retention time of compound $\mathrm{g}$ was the same as that of 8-heptadecene (from extracts of $P$. peltifer, see "Methods and Materials"). Compound $\mathrm{j}(\mathrm{RT}=19.27 \mathrm{~min})$, also a minor component, had the following mass spectrum: $m / z 264$ (11), 235 (3), 221 (4), 207 (4), 165 (4), 151 (7), 137 (10), 123 (10), 109 (22), 95 (61), 81 (16), 79 (52), 67 (100), 55 (38), 54 (32), 44 (32), and 41 (69). The compound was tentatively identified as 9,17-octadecadienal on the basis of these mass spectral data.

The pattern of the ten compounds was consistently detected in 30 of the 35 individuals of adult $O$. berlese $i$ (Fig. 1a), with relatively low inter-individual variation with respect to the relative abundance of components (data not shown). The other five extracts did not contain any of the components.

Extracts of Juvenile O. berlesei Extracts of juveniles (exclusively deuto- and tritonymphs) contained only two compounds in a 1:2 ratio (peak $\mathrm{c}_{1}$ and $\mathrm{c}$ in Fig. 1b): The major component $\mathrm{c}$ corresponded to tentatively identified $(3 S, 8 S)$-chrysomelidial from the adult extracts, based on identical retention time and mass spectrum. The minor component $\mathrm{c}_{1}$ eluted earlier ( $\mathrm{RT}=13.32 \mathrm{~min}$ ) but was not fully separable from $(3 S, 8 S)$-chrysomelidial. The mass spectrum of compound $\mathrm{c}_{1}$ appeared to be similar to that of (3S,8S)-chrysomelidial (Fig. 2); one difference, however, was the greater intensity of the ion at $m / z 138$ in relation to the ion at $m / z 148$ [c.f., in (3S,8S)-chryomelidial the ion at $m / z 148$ is more intense]. These data suggested the compound to be one of the other possible stereoisomers of chrysomelidial, most likely an epimeric diastereomer of $(3 S, 8 S)$-chrysomelidial. Moreover, the retention time and mass spectrum of compound $\mathrm{c}_{1}$ fully corresponded with those of the earliereluting peak of the synthetic chrysomelidial mixture, limiting the possibilities to epi-chrysomelidial $(3 S, 8 R$ chrysomelidial) and its enantiomer, $(3 R, 8 S)$-dehydroiridodial. In addition, its retention time and mass spectrum matched those of plant-derived $(3 R, 8 S)$-dehydroiridodial. Thus, again assuming that dehydroiridodials are exclusively plant-derived compounds, compound $\mathrm{c}_{1}$ was tentatively identified as epi-chrysomelidial ( $3 S, 8 R$-chrysomelidial).

From a total of eight extracts of juvenile $O$. berlesei investigated (derived from 18 individuals), seven extracts consistently showed these two stereoisomers only; the other extract (an extract of a single individual) apparently did not contain any compounds at all.

Potential Secretory Glands The most conspicuous exocrine gland system of $O$. berlesei is the paired opisthonotal glands that, in light microscopic observations, are visible through the cuticle of the notogaster as kidney-shaped sacs of considerable size (maximal diameter about $0.5 \mathrm{~mm}$ : i.e., ca. 1/3 of body length; see Fig. 3b). The glands open to the notogastral surface via one single flap-provided pore orifice on either side of the body (Fig. 3a,c,d). The flap,

Fig. 3 Topography and external morphology of opisthonotal glands in Oribotritia berlesei. a Scanning electron micrograph (SEM) of an adult individual, lateral view. The arrow marks the orifice of the left opisthonotal gland. b Opisthonotal glands are visible through the integument under light microscopy. c SEM of an opisthonotal gland pore with flap open. d SEM of the pore of another individual,
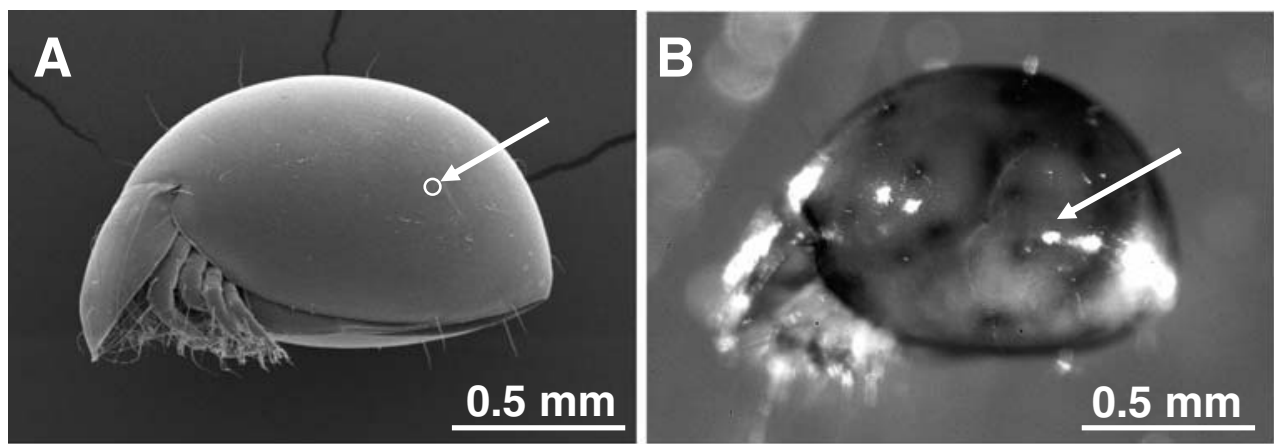
orifice closed
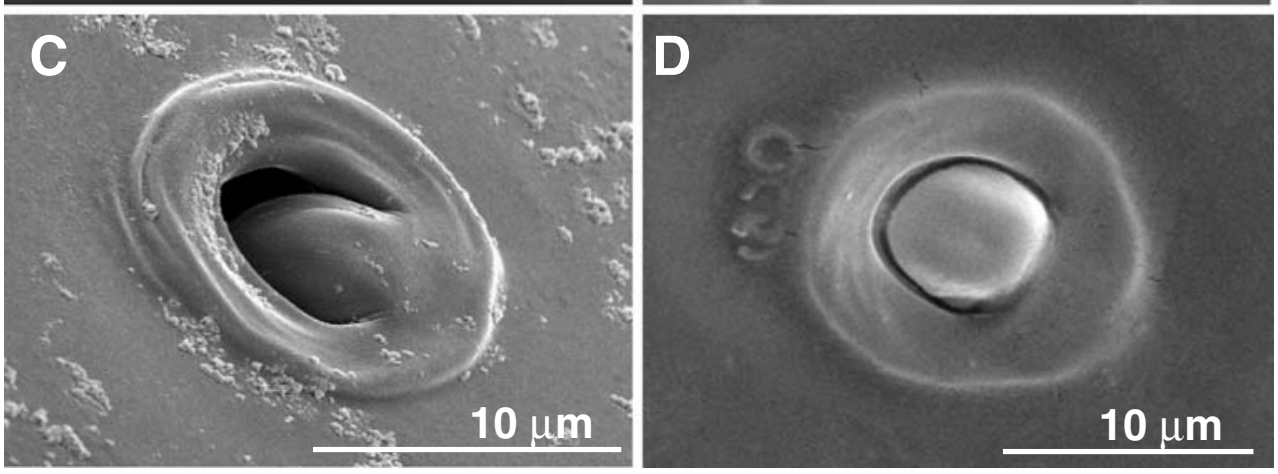
presumably an external closing mechanism, was found either in an opened (Fig. 3c) or a closed position (Fig. 3d) in different individuals. No other comparably large glands or pore orifices are present in this species. Opisthonotal glands are present in adults and in all juvenile stages.

\section{Discussion}

Occurrence of Chrysomelidial Even though iridoid monoterpenes are widely distributed in exocrine secretions of a diverse range of arthropods, chrysomelidial, so far, has only been reported from leaf beetle larvae (Chrysomelidae) and from certain rove beetles (e.g., Pasteels et al. 1982; Weibel et al. 2001). Originally, chrysomelidial was found as a major constituent of the larval eversible glands of the chrysomelids, Gastrophysa cyanea (Blum et al. 1978) and Plagiodera versicolora (Meinwald et al. 1977), and its stereochemistry later elucidated by Meinwald and Jones (1978). According to these studies, and to current knowledge, chrysomelidial in leaf beetles is a mixture of two diastereomers, $(3 S, 8 S-$ chrysomelidial (= the original chrysomelidial) and $(3 S, 8 R)$ chrysomelidial (= epi-chysomelidial), with the former being the predominant isomer in the majority of chrysomelidialproducing species (e.g., Sugawara et al. 1979; Pasteels et al. 1982). In contrast, the remaining two possible stereoisomers (there are four stereoisomers due to the two asymmetric carbon atoms at $\mathrm{C}_{3}$ and $\mathrm{C}_{8}$ ) are termed "dehydroiridodials" and are considered of plant origin only (Yoshihara et al. 1978; Sugawara et al. 1979). Under the conditions used in this study, we were able to separate the diastereomers but not the respective enantiomers of chrysomelidial and dehydroiridodial. Assignments of chrysomelidials and dehydroiridodials are often misinterpreted in the literature. For instance, Bellesia et al. (1986) described the chrysomelidials as a pair of enantiomers, whereas they are in fact diastereomers. In the present paper, we use the classification of Sugawara et al. (1979) for these compounds, but also use the more common nomenclature of Bellesia et al. (1986). Thus, assuming dehydroiridodials are found exclusively in plants, the compounds in $O$. berlesei extracts are $(3 S, 8 S)$-chrysomelidial and $(3 S, 8 R)$-chrysomelidial (epi-chrysomelidial).

Our analysis of $O$. berlesei is not only the first example of the chemistry of exocrine glands of Euphthiracaroidea but also the only report of chrysomelidials found outside the Coleoptera. In $O$. berlesei, chrysomelidials most likely originate from the opisthonotal glands: these glands are quite large in $O$. berlesei, and thus probably capable of producing compounds in the amount detected. Furthermore, some of the components, especially 6,9-heptadecadiene and some other hydrocarbons, found in the extract are characteristic constituents of opisthonotal glands of many oribatid (and astigmatid) mite species (Kuwahara 2004; Raspotnig
2006). Functionally, at least in Chrysomelidae, iridoid monoterpenes such as chrysomelidials are considered defensive or for microbial protection; these functions are also generally attributed to opisthonotal glands of Oribatida (Raspotnig et al. 2003).

Chemosystematic Impact With respect to chemosystematic studies that use opisthonotal gland profiles of Oribatida, the finding of chrysomelidials in $O$. berlesei is significant. In preliminary investigations, chrysomelidials have been found also in other species of Euphthiracaroidea (data unpublished), suggesting that this chemical class may be characteristic for species of Oribotritiidae, possibly representing an important chemical "synapomorphy" of this family. Also, two further compounds in extracts of $O$. berlesei, if our tentative identifications are correct, would be novel for oribatid and astigmatid mite opisthonotal gland secretions. The diterpene, $\beta$-springene, which we tentatively identified as one of the three main components of the secretion, was originally found in secretions of springboks (Burger et al. 1978), and has also been detected in exocrine secretions of further vertebrates, especially mammals and reptiles (e.g., Waterhouse et al. 1996; Schulz et al. 2003), and invertebrates such as certain hymenopterans (e.g., Howard et al. 2003; Cruz-Lopez et al. 2005), but not in arachnids. The other novel compound (for oribatid and astigmatid mite opisthonotal gland secretions) is the tentatively identified 9,17-octadecadienal, a minor constituent of the $O$. berlese $i$ extract. Both tentatively identified compounds, $\beta$-springene and 9,17-octadecadienal, may be of chemosystematic value in Oribatida. Their unequivocal identification and possible distribution across other species of Euphthiracaroidea remain to be studied.

Astigmatid Compounds Perhaps the most surprising result of our study is the lack of "astigmatid compounds" in the opisthonotal gland extract of $O$. berlesei. These compounds are common secretion constituents in MixonomataDesmonomata and Astigmata, and are regarded as characteristic of all groups above middle-derived mixonomatans. In Collohmanniidae (proposed close relatives of euphthiracaroids), astigmatid compounds dominate opisthonotal gland secretions. Astigmatid compounds, at least in some higher-derived desmonomatans and in brachypylines, may be reduced and replaced by other (novel) compounds in adult secretions of certain groups as in, for example, Hermannia convexa (Raspotnig et al. 2005a) and Scheloribates ssp. (Takada et al. 2005). Thus, there is a chemical dimorphism between juveniles and adults, with juvenile secretions being distinguished by the plesiomorphic, astigmatid compounds-rich condition. This chemical dimorphism was tested in $O$. berlesei; however, neither adults nor juveniles contained any astigmatid compounds. 
Even though the lack of astigmatid compounds in $O$. berlesei may be explained by complete evolutionary reduction, this situation may suggest a possible polyphyletic origin of astigmatid compounds in opisthonotal glands in general. The lack of astigmatid compounds is even more surprising in light of the classification system proposed by Haumann (1991), in which oribotritiids are regarded as basal euphthiracaroids. Astigmatid compounds-free opisthonotal gland secretions in adults and juveniles also occur in several groups of brachypylines (Raspotnig, unpublished data), but these groups are considered highly derived. The lack of astigmatid compounds in Oribotritiidae could be reconciled by a systematic replacement of euphthiracaroids below the Collohmanniidae, corresponding to a possible split from the oribatid stem-line before astigmatid compounds evolved. However, this is not supported by morphological characters (Grandjean 1969). The lack of astigmatid compounds may not necessarily be true for all Oribotritiidae, and any extrapolation from data for $O$. berlesei remains speculative. Our major aim for future studies is the search for astigmatid compounds in other representatives of euphthiracaroid families.

Acknowledgments This work was supported by a grant from the Austrian Science Fund (FWF), project number P18486. We thank Dr. Günther Krisper (Institute of Zoology, Karl-Franzens-University, Graz, Austria) for his help in the determination and discrimination of Oribotritia species.

Open Access This article is distributed under the terms of the Creative Commons Attribution Noncommercial License which permits any noncommercial use, distribution, and reproduction in any medium, provided the original author(s) and source are credited.

\section{References}

Bellesia, F., Guelfi, F., Pagnoni, U. M., and PinetTi, A. 1986. The base-catalyzed cyclization of 10-oxocitral. Synthesis of chrysomelidial and dehydroiridodial. Tetrahedron Lett. 27:381-382.

Blum, M. S., Wallace, J. B., Duffield, R. M., Brand, J. M., FALES, H. M., and SOKOLOSKI, E. A. 1978. Chrysomelidial in the defensive secretion of the leaf beetle Gastrophysa cyanea Melsheimer. J. Chem. Ecol. 4:47-53.

Burger, B. V., Le Roux, M., Spies, H. S. C., Truter, V., and BIGALKE, R. C. 1978. Mammalian pheromone studies. III. (E,E)7,11,15-Trimethyl-3-methylenehexadeca-1,6,10,14-tetraene, a new diterpene analogue of $\mathrm{b}$-farnesene from the dorsal gland of the springbok, Antidorcas marsupialis. Tetrahedron Lett. 52:5221-5224.

Cruz-Lopez, L., Malo, E. A., Morgan, E. D., Rincon, M., GUZMAN, M., and ROJAS, J. C. 2005. Mandibular gland secretion of Melipona beecheii: chemistry and behavior. J. Chem. Ecol. 31:1621-1632.

Domes, K., Althammer, M., Norton, R. A., Scheu, S., and MARAUN, M. 2007. The phylogenetic relationship between Astigmata and Oribatida (Acari) as indicated by molecular markers. Exp. Appl. Acarol. 42:159-171.
GrandjeAn, F. 1969. Considerations sur le classement des Oribates. Leur division en 6 groupes majeurs. I. Les affinites de Collohmannia gigantea Sellnick, 1922. Acarologia 11:127-153.

HAUMANN, G. 1991. Zur Phylogenie primitiver Oribatiden (Acari: Oribatida). DBV-Verlag, Graz.

Howard, R. W., BAKer, J. E., and Morgan, E. D. 2003. Novel diterpenoids and hydrocarbons in the Dufour gland of the ectoparasitoid Habrobracon hebetor (Say) (Hymenoptera: Braconidae). Arch. Insect Biochem. Physiol. 54:95-109.

Kuwahara, Y. 2004. Chemical ecology of astigmatid mites, pp. 76 109, in R. T. Cardé, and J. G. Millar (eds.). Advances in Insect Chemical EcologyCambridge University Press, Cambridge.

MEINWALD, J., and JONES, T. H. 1978. Synthesis and stereochemistry of chrysomelidial and plagiolactone. J. Am. Chem. Soc. 100:1883-1886.

Meinwald, J., Jones, T. H., EISNER, T., and HicKs, K. 1977. New methylcyclopentanoid terpenes from larval defensive secretion of a chrysomelid beetle (Plagiodera versicolora). Proc. Natl. Acad. Sci. U. S. A. 74:2189-2193.

Murell, A., Dobson, S. J., Walter, D. E., Campbell, N. J. H., SHAO, R., and BARKER, S. C. 2005. Relationships among the three major lineages of the Acari (Arthropoda: Arachnida) inferred from small subunit rRNA: paraphyly of the Parasitiformes with respect to the Opilioacariformes and relative rates of nucleotide substitution. Invertebrate Systematics. 19:383-389.

NoRTON, R. A. 1998. Morphological evidence for the evolutionary origin of Astigmata. Exp. Appl. Acarol. 22:559-594.

Oldham, N. J., Veith, M., Boland, W., and Dettner, K. 1996. Iridoid monoterpene biosynthesis in insects: evidence for a de novo pathway occurring in the defensive glands of Phaedon armoraciae (Chrysomelidae) leaf beetle larvae. Naturwissenschaften 83:470 473.

Pasteels, J. M., Breakman, J.-C., Daloze, D., and Ottinger, R. 1982. Chemical defence in chrysomelid larvae and adults. Tetrahedron 38:1891-1897.

RASPOTNIG, G. 2006. Characterisation of monophyletic oribatid groups by oil gland chemistry - a novel systematic approach in Oribatida (Acari). Abh. Ber. Naturkundemus. Görlitz 78:31-46.

Raspotnig, G., Schuster, R., Krisper, G., Fauler, G., and Leis, H. J. 2001. Chemistry of the oil gland secretion of Collohmannia gigantea (Acari: Oribatida). Exp. Appl. Acarol. 25:933-946.

Raspotnig, G., SchUSter, R., and KrisPer, G. 2003. Functional anatomy of oil glands in Collohmannia gigantea (Acari, Oribatida). Zoomorpholgy 122:105-112.

RASPOTNIG, G., KRISPER, G., and SCHUSTER, R. 2005a. Ontogenetic changes in the chemistry and morphology of oil glands in Hermannia convexa (Acari: Oribatida). Exp. Appl. Acarol. 35:47-58.

Raspotnig, G., Krisper, G., Schuster, R., Fauler, G., and Leis, H. J. 2005b. Volatile exudates from the oribatid mite, Platynothrus peltifer. J. Chem. Ecol. 31:419-430.

SAKATA, T., and NORTON, R. A. 2001. Opisthonotal gland chemistry of early-derivative oribatid mites (Acari) and its relevance to systematic relationships of Astigmata. Int. J. Acarol. 27:281-291.

SAKATA, T., and NORTON, R. A. 2003. Opisthonotal gland chemistry of a middle-derivative oribatid mite, Archegozetes longisetosus (Acari: Trhypochthoniidae). Int. J. Acarol. 29:345-350.

SAKatA, T., TAGAMI, K., and KUWAHARA, Y. 1995. Chemical ecology of oribatid mites. I. Oil gland components of Hydronothrus crispus Aoki. J. Acarol. Soc. Jpn. 4:69-75.

Sakata, T., Shimano, S., and Kuwahara, Y. 2003. Chemical ecology of oribatid mites. III. Chemical composition of oil gland exudates from Trhypochthoniellus sp. and Trhypochthonius japonicus (Acari: Trhypochthoniidae). Exp. Appl. Acarol. 29:279-291.

Saporito, R., Donelly, M. A., Norton, R. A., Garaffo, H. M., SPANDE, T. F., and DALY, J. W. 2007. Oribatid mites as a major 
source for alkaloids in poison frogs. Proc. Natl. Acad. Sci. U. S. A. 104:8885-8890.

SchulZ, S., KRÜCKert, K., and Weldon, P. J. 2003. New terpene hydrocarbons from the Alligatoridae (Crocodylia, Reptilia). $J$. Nat. Prod. 66:34-38.

Shimano, S., SAKata, T., Mizutani, Y., Kuwahara, Y., and AOKI, J.-I. 2002. Geranial: the alarm pheromone in the nymphal stage of the oribatid mite Nothrus palustris. J. Chem. Ecol. 28:1831-1837.

Sugawara, F., Matsuda, K., Kobayashi, A., and Yamashita, K. 1979. Defensive secretion of chrysomelid larvae Gastrophysa atrocyanea Motschulsky and Phaedon brassicae Baly. J. Chem. Ecol. 5:635-641.

TAKAdA, W., SAKata, T., Shimano, S., EnAmi, Y., Mori, N., NishidA, R., and KUWAHARA, Y. 2005. Scheloribatid mites as the source of pumiliotoxins in dendrobatid frogs. J. Chem. Ecol. 31:2403-2415.
Vincenti, M., Guglielmetti, G., Cassani, G., and Tonini, C. 1987. Determination of double bond position in diunsaturated compounds by mass spectrometry of dimethyl disulfide derivatives. Anal. Chem. 59:694-699.

Waterhouse, J. S., Ke, J., Pickett, J. A., and Weldon, P. J. 1996. Volatile components in the dorsal gland secretion of the collared peccary, Tayassu tajacu (Tayassuidae, Mammalia). J. Chem. Ecol. 22:1307-1314.

Weibel, D. B., Oldham, N. J., Feld, B., Glombitza, G., Dettner, K., and BOLAND, W. 2001. Iridoid biosynthesis in staphylinid rove beetles (Coleoptera: Staphylinidae, Philonthinae). Insect Biochem. Mol. Biol. 31:583-591.

YoshiHARA, K., SAKAI, T., and SAKAN, T. 1978. Dehydroiridodial, the pungent principle of Actinidia polygama Miq. Chem. Lett. 1978:433-434. 